\title{
CARACTERIZACIÓN BIOQUÍMICA DE LAS ABEJAS NATIVAS DE DOS ECOSISTEMAS DISTINTOS DE LA SELVA CENTRAL DEL PERÚ
}

\section{BIOCHEMICAL CHARACTERIZATION OF THE NATIVE BEES OF TWO DIFFERENT ECOSYSTEMS FROM THE CENTRAL FOREST OF PERU}

\author{
Eleazar Pérez Castro', Raúl Yaranga Cano', Carina Escurra Dávila², \\ Javier Quezada Euán ${ }^{3}$, Amano Kasuhiro ${ }^{4}$, Octavio Macias Macias ${ }^{5}$
}

\section{RESUMEN}

Con la finalidad de caracterizar en forma bioquímica las abejas nativas de los ecosistemas de San Ramón y Satipo de la Selva Central del Perú, se ha elegido un área de $1000 \mathrm{~m}^{2}$ en cada uno de ellos, para identificar los nidos presentes y colectar muestras de abejas prontas a nacer y de la misma edad en frascos de $50 \mathrm{ml}$ conteniendo alcohol al 99 $\%$. El trabajo se inició en abril de 2004 y concluyó en marzo de 2005. El análisis bioquímico de basó en la tecnología de Kloth (1992) para proteínas en sistema Disc-Page. Se ha encontrado un total de 21 nidos, siete en San Ramón (tres Meliponas, tres Trigonas, una Plebeia) y 14 en Satipo (Siete Meliponas, cinco Trigonas, dos Plebeias). Se seleccionó al azar para las corridas electroforéticas, tres muestras del ecosistema de San Ramón (una de Melipona, una de Trigona y una de Plebeia) y seis muestras del ecosistema de Satipo (dos de Melipona, dos de Trigona y dos de Plebeia). Se concluye que los individuos de los géneros de abejas nativas de ambos ecosistemas podrían corresponder a una misma especie, por las similares características que presentan las bandas de proteínas después de la electroforesis. Los resultados muestran que la metodología seleccionada para caracterización proteica de abejas nativas, permite la separación de bandas nítidas en genotipos de Melipona, lo que constituye una herramienta que puede ser utilizada en posteriores trabajos de caracterización genética.

Palabras clave: Abeja nativa, caracterización, Selva Central, Melipona, Trigona, Plebeya.

\begin{abstract}
With the purpose of characterizing in biochemical form the native bees of the ecosystems of San Ramon and Satipo of the Central Forest of the Peru. $1000 \mathrm{~m}^{2}$ has been chosen in each one of them to identify the present nests and to collect samples of quick bees to be born and of the same age in bottles of 50 mililiter containing alcohol to $99 \%$. The work initiated in April of 2004 and concluded in March of 2005. The biochemical analysis development with technologic of Kloth (1992) for proteins in DiscPAGE system. Has been a total of 21 nests of native bees, seven in San Ramon (three Meliponas, three Trigonas, one Plebeia) and 14 in Satipo (Seven Meliponas, five Trigonas, two Plebeias). Itself selected at random for the electroforetic bullfights, three samples of the ecosystem of San Ramon (one of Melipona, one of Trigona and one of Plebeia) and six samples of the ecosystem of Satipo (two of Melipona, two of Trigona and two of Plebeia). we concluded that the individuals of the generous the native bees of both ecosystems, they would be able to correspond to a same species, by the similar characteristics that present the bands of proteins after the electrophoresis. The results show that methodology selected for protein characterization of native bees, allows the separation of bands in genotypes of Melipona, which constitutes a tool that can be used in later works of genetic characterization.
\end{abstract}

Key words: Native bee, characterization, Central Forest, Melipona, Trigona, Plebeia.

Facultad de Zootecnia. Universidad Nacional del Centro del Perú.

CEPIDA.

${ }^{3}$ UADY, México.

${ }^{4}$ NILGC,Japón.

Univ. de Guadalajara, México. 


\section{INTRODUCCIÓN}

Los principales géneros de abejas nativas o abejas sin aguijón de importancia económica para el hombre son: Melipona y Trigona. Las Trigonas habitan en todas las áreas tropicales y subtropicales del Mundo, en tanto Melipona se encuentra únicamente en el Neotrópico (Ayala et al., 1996). Las abejas nativas están distribuidas desde el norte de México hasta el norte de Argentina y actualmente se ven amenazadas por múltiples factores (Imperatriz -Fonseca, 1989) y se hace necesario pensar en acciones para preservarlas, una de las cuales es su explotación racional, para ello es necesario caracterizarlas y tener conocimiento de su biología. El problema central de la investigación se basa en que en nuestro país no se ha llevado a cabo trabajos de caracterización bioquímica de las abejas nativas y se hace necesaria la estandarización de técnicas apropiadas para caracterizar los diferentes géneros y especies. El presente trabajo tiene como objetivos: determinar la variabilidad proteica de las abejas nativas de los ecosistemas de San Ramón y Satipo en la Selva Central del Perú; determinar la mejor resolución y polimorfismo del método de electroforesis para un género de abejas nativas; y describir la biodiversidad de los géneros de abejas nativas.

\section{MATERIAL Y MÉTODOS}

La investigación se realizó durante el periodo comprendido de abril de 2004 a marzo de 2005, con muestras de abejas nativas colectadas en la E.E.A. San Ramón y E.E.A. Satipo de la UNCP que se encuentran ubicadas en el distrito de San Ramón, Chanchamayo ( $850 \mathrm{msnm}$ ) y en el distrito de Satipo, Satipo (550 msnm) respectivamente de la región Junín. El muestreo se realizó en un área de 1000 metros cuadrados en cada ecosistema con el fin de localizar los nidos y de cada nido se colectaron varios individuos prontos a nacer y de la misma edad en frascos de $50 \mathrm{ml}$ conteniendo alcohol al 99 $\%$.

La entrada del nido, materiales de construcción y comportamiento defensivo fueron las

características que han permitido determinar el género correspondiente de cada muestra de abejas.

El análisis electroforético de proteínas se desarrolló en el Laboratorio de Biotecnología de la Universidad Nacional del Centro del Perú. Se compararon tres géneros de abejas nativas (Melipona, Trigona $y$ Plebeia). Para la extracción de proteínas se utilizó el tórax de la abeja y la técnica de Kloth (1992). Luego se procedió a cargar las muestras tomando $200 \mu \mathrm{l}$ del extracto, agregando $50 \mu \mathrm{l}$ de sacarosa al $60 \%$ y $5 \mu \mathrm{l}$ de amido black agitando hasta homogeneizar la muestra. Para la corrida (tres horas) se tomó $10 \mu \mathrm{l}$ de la muestra cargada. Se usaron geles de poliacrilamida (5 $15 \%$ ), en un sistema Disc-PAGE con cámara de electroforesis vertical $(10 \times 8 \mathrm{~cm})$ y las siguientes condiciones de corrida: 1). voltaje inicial $50 \mathrm{v}$ el cual, se incrementó después de $15-20 \mathrm{~min}$ a $100 \mathrm{v}$, amperaje y tiempo de corrida variable; 2 ). amperaje fijo a $30 \mathrm{~mA}$ y voltaje libre, el cual al transcurrir el tiempo de corrida se estabilizó en 100 v.

La visualización de proteínas se realizó utilizando Azul de Coomassie como indicador. La corrida electroforética fue con la finalidad de obtener un patrón de bandeo adecuado que permitiera la caracterización de uno o más genotipos de abejas en cada género. El parámetro evaluado fue la resolución y el polimorfismo. Luego se describió la biodiversidad de abejas nativas existentes a nivel de género.

\section{RESULTADOS}

Se han colectado 21 muestras de abejas (siete de San Ramón y 14 de Satipo). Se seleccionó al azar para las corridas electroforéticas, tres muestras del ecosistema de San Ramón (una de Melipona, una de Trigona y una de Plebeia) y seis muestras del ecosistema de Satipo (dos de Melipona, dos de Trigona y dos de Plebeia). Los individuos de cada uno de los géneros de abejas nativas (Melipona, Trigona y Plebeya) de los ecosistemas de San Ramón y Satipo, podrían corresponder a una misma especie, por las similares características que presentan las bandas de proteínas después de la electroforesis. Los zimotipos de proteínas en DiscPAGE $515 \%$ para extractos de Melipona permitió la separación de bandas nítidas, lo que constituye una herramienta que puede ser utilizada en posteriores trabajos de caracterización genética.

El patrón de bandeo del extracto de Trigona no permitió una buena separación de bandas, ya que se formó al final del carril una banda gruesa y marcada. En las muestras de Plebeia no se visualiza claramente las proteínas, observándose bandas muy tenues, lo que permitió inferir que el tampón de extracción no es adecuado para extraer proteínas en tejidos de Plebeia.

Los resultados muestran que la metodología seleccionada permite la separación de bandas de proteínas en genotipos de Melipona. Los resultados de caracterización proteica, así como la entrada del nido, materiales de construcción y comportamiento defensivo, permiten inferir que la biodiversidad de abejas nativas del ecosistema de San Ramón y el ecosistema de Satipo, dentro de géneros son las mismas, por las similares características que presentan cada una; por lo tanto, no existe variación en forma morfológica o bioquímica y se sugiere que la descripción de las abejas nativas de los géneros Melipona, Trigona y Plebeia para ambos 
ecosistemas sean las mismas. Según la observación de los individuos y la entrada del nido, antes de colectar las muestras de abejas, se describe las siguientes características:

\begin{abstract}
Abejas nativas del género Melipona. Las abejas nativas del género Melipona son de tamaño medio, con longitud del cuerpo entre 9,7 a 10,7 mm, de color gris en el tórax y terguitos metasomales negros con franjas amarillas; terguitos con pubescencia abundante, corta, anaranjada o amarilla; las patas son de color café y negro. En estado silvestre las abejas del género Melipona habita casi exclusivamente en cavidades de árboles de al menos 20 a $25 \mathrm{~cm}$ de diámetro y de 2,5 a $3 \mathrm{~cm}$ de grosor en las paredes de la cavidad y a una distancia de 1 ó $2 \mathrm{~m}$ hasta $20 \mathrm{~m}$ sobre el nivel del suelo. El nido es construido con una mezcla de la cera que producen las abejas con resina y goma vegetales Ilamado cerumen. La entrada del nido consiste generalmente de un simple agujero con un borde ligeramente saliente y a veces con un poco de barro a su alrededor en forma radiada o de estrella.
\end{abstract}

\begin{abstract}
Abejas nativas del género Trigona. Las abejas nativas del género Trigona no poseen aguijón, son de color negro con las puntas de las alas terminadas en color y son sumamente dóciles. En estado silvestre se encuentra en troncos de árboles, cavidades de rocas y muros de concreto. El cerumen es una mezcla de color café hecha de la cera de las abejas con propóleos que se utiliza para la construcción del nido y dependiendo de las estructuras internas del mismo, pueden ser cerumen suave o duro. El cerumen viejo es quebradizo y el nuevo es suave. La entrada al nido tiene una prolongación hacia el exterior que se ensancha gradualmente en forma de trompeta, esta hecha de cerumen oscuro y que también se prolonga hasta las celdas de cría formando el tubo interno de la entrada.
\end{abstract}

Abejas nativas del género Plebeia. Género de abejas diminutas que son atraídos por la transpiración humana se caracterizan por sus pequeñas dimensiones corporales, generalmente inferior a $4 \mathrm{~mm}$, también son llamadas abejas mosquito, de coloración negra. La entrada del nido es poco reluciente y esta construido de un tubo fino de cera amarilla en la entrada de su colmena. Los nidos se pueden ubicar en huecos de árboles o cualquier otra cavidad.

\section{DISCUSIÓN}

Las muestras correspondieron a individuos prontos a nacer y fueron colectados directamente de los panales de cría para garantizar un similar estado fisiológico y ontogenético. El material seleccionado para preparar los extractos de las muestras fue el tórax de las abejas nativas, ya que con esta estructura del cuerpo se logra mayor resolución y polimorfismo que con el resto de tejidos de otras partes del cuerpo. Aunque Blogg e Imprie (1982), señalan que ciertos tejidos son metabólicamente más estables que otros usados para realizar electroforesis de proteínas, y que cualquiera que sea el tejido seleccionado como fuente para preparar extractos, debe garantizar que las muestras se encuentren en el mismo estado fisiológico y ontogenético, ya que está ampliamente demostrado que ocurren cambios cualitativos y cuantitativos en proteínas con la edad del animal.

La separación de bandas nítidas, constituye una herramienta que puede ser utilizada en posteriores trabajos de caracterización genética (Blogg e Imprie 1982) de abejas nativas, particularmente del género Melipona. Los resultados de caracterización proteica, así como la entrada del nido, materiales de construcción y comportamiento defensivo (Ayala et al., 1996), han permitido concluir que la biodiversidad de abejas nativas del ecosistema de San Ramón y el ecosistema de Satipo, dentro de géneros son las mismas, por las similares características que presentan cada una; por lo tanto, no existe variación en forma morfológica o bioquímica y se sugiere que la descripción de las abejas nativas (Imperatriz -Fonseca, 1989) de los géneros Melipona, Trigona y Plebeia para ambos ecosistemas sean las mismas.

Las abejas nativas Melipona, Trigona y Plebeia del ecosistema de San Ramón comparado con los del ecosistema de Satipo, dentro de géneros son las mismas, por las similares características que presentan las bandas de proteínas después de la electroforesis. La metodología seleccionada para caracterización proteica de las abejas nativas, permite la separación de bandas nítidas en genotipos de Melipona, lo que constituye una herramienta que puede ser utilizada en posteriores trabajos de caracterización genética y se sugiere una sola descripción para cada género de abejas nativas de la Selva Central del Perú. 


\section{REFERENCIAS BIBLIOGRÁFICAS}

1. Ayala R, Griswold T, Vanega D. Apoidea (Hymenoptera). En: Llorente; 1996.

2. García AN, González ES. Biodiversidad, Taxonomía y Biogeografía de Artrópodos de México: Hacia una síntesis de su conocimiento. CONABIO-UNAM. pp. 423-464.

3. Fonseca I. The development of Meliponiculture in Brazil. XXXII International Congress of Apiculture. Rio de Janeiro: 1989. Brazil. 22-28. PP66-8.

4. Kloth R. Variability of Malate Dehydrogenase among cotton cultivars with differing fiber trains. Crp. 1992.Science 32:61 7-620.

5. Blogg DT, Imprie J. Starch gel electrophoresis for soybean cultivar identification seed. 1982. Sci. \& Techno 10:19-24.

E-mail: aleazarperu@yahoo.com 